\title{
Predicting blood pressure in aortas treated for coarctation
}

\author{
D. Pathirana ${ }^{1}$ \\ B. M. Johnston ${ }^{2}$ \\ P. R. Johnston ${ }^{3}$
}

(Received 22 December 2015; revised 12 May 2016)

\begin{abstract}
Coarctation of the aorta is a narrowing of the aorta that is present at birth. Treatments for coarctation of the aorta affect the aorta wall stiffness. This study uses a 1D model to predict the effects of altered wall stiffness. Stent treatments increase both blood pressure and the radial change of the aorta wall over a heartbeat cycle, compared to both healthy aortas and the resection and end-to-end anastomosis treatment.
\end{abstract}

http://journal.austms.org.au/ojs/index.php/ANZIAMJ/article/view/10388 gives this article, (C) Austral. Mathematical Soc. 2016. Published June 7, 2016, as part of the Proceedings of the 12th Biennial Engineering Mathematics and Applications Conference. ISSN 1446-8735. (Print two pages per sheet of paper.) Copies of this article must not be made otherwise available on the internet; instead link directly to this URL for this article. 


\section{Contents}

1 Introduction

C33

2 Model outline

2.1 Parameter values . . . . . . . . . . . . . . . . C C38

2.2 Stiffness . . . . . . . . . . . . . . . . . C38

2.3 Numerical method . . . . . . . . . . . . . . C41

3 Results and discussion $\quad$ C43

3.1 Pressure changes . . . . . . . . . . . . . . C C43

3.2 Radial changes . . . . . . . . . . . . . . . C44

4 Conclusion

C46

References

C48

\section{Introduction}

Coarctation of the aorta ( $\mathrm{COA})$ is a congenital heart defect affecting newborn babies. In the developing foetus, oxygenated blood is supplied by the mother, so the lungs of the foetus are bypassed with an artery, called the ductus arteriosus, that connects the pulmonary artery (that normally routes blood to the lungs) to the aorta (Figure 1). After childbirth, the newborn needs to oxygenate blood, hence the ductus arteriosus closes. The prevailing theory for the cause of $\mathrm{COA}$ is that when the ductus arteriosus closes, part of the aorta narrows as well (Figure 2) [1, 2].

$\mathrm{CoA}$ is a disease characterised by a narrowed region of the aorta, and accounts for $5-8 \%$ of congenital heart defects, or between three and four in every 10000 live births [3, 4]. It is considered a serious disease with a mean age of mortality of 35 years [5]. Coarctations generally occur after the arteries that supply blood to the upper body diverge from the aorta, hence severe 
Figure 1: Schematics of the heart and surrounding vasculature with a healthy aorta before birth, when the ductus arteriosus is open.

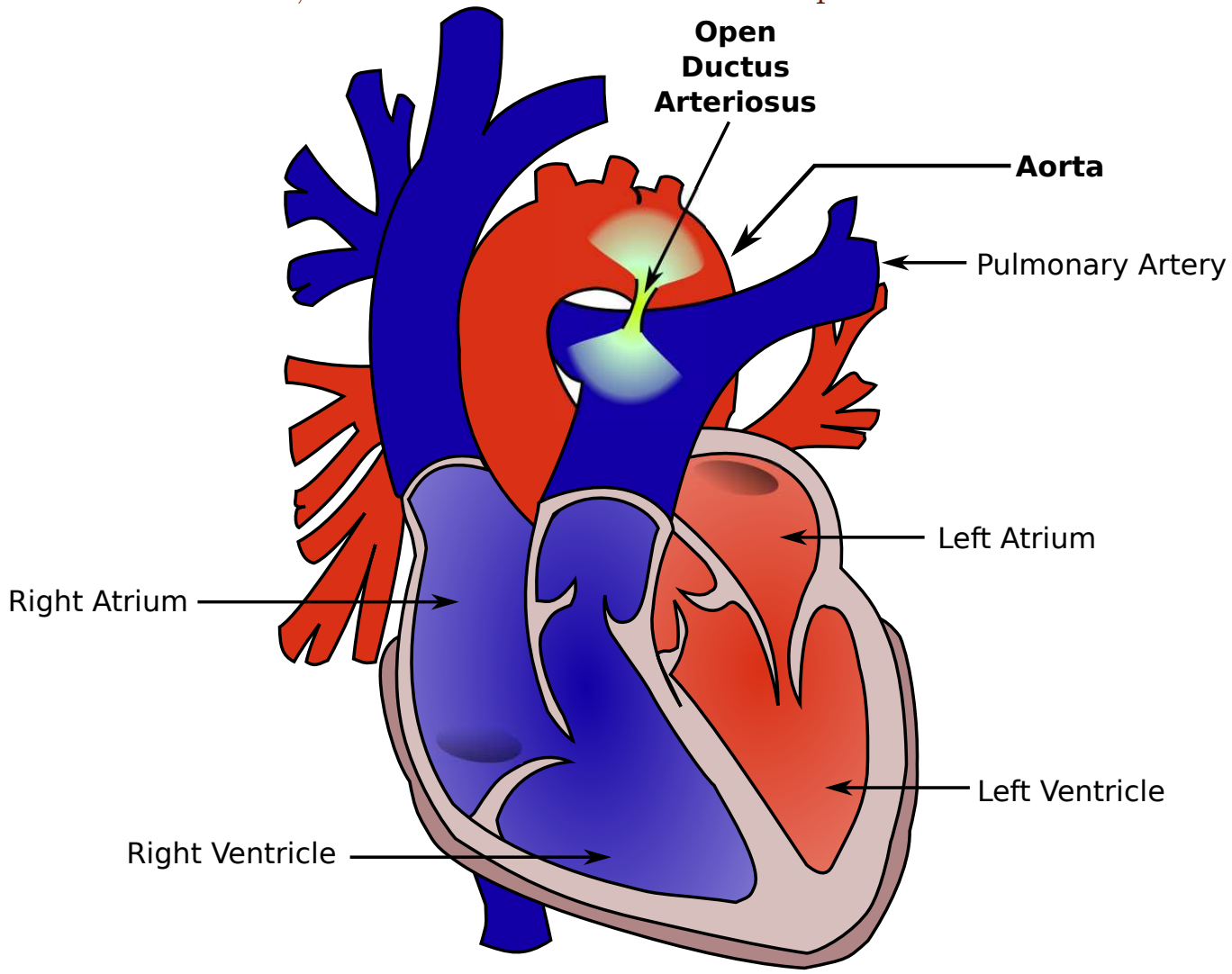


Figure 2: Schematics of the heart and surrounding vasculature with an aorta after birth and closure of the ductus arteriosus, featuring a coarctation.

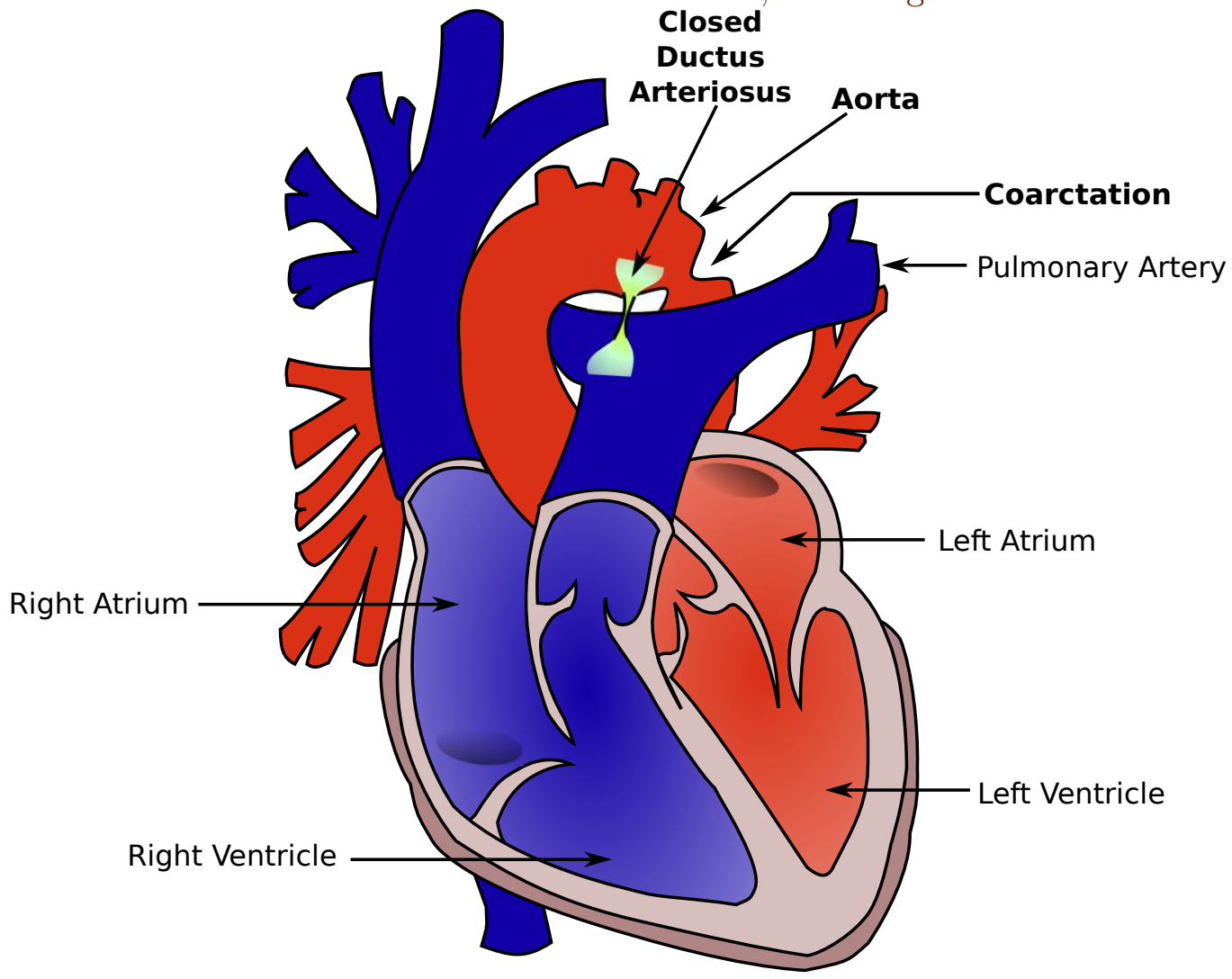


coarctations may result in a lack of blood flow to the lower regions of the body. Several treatments that extend the lifespan of patients are available; however, treated patients still experience decreased life expectancies compared to healthy populations [3].

Common surgical treatments include: left subclavian flap aortoplasty, where a section of the artery that supplies blood to the left arm is used as a patch to expand the coarctated region; tubular bypass graft, where the coarctated region is bypassed by attaching a tube above and below the coarctation; and resection and end-to-end anastomosis, where the coarctated region of the aorta is cut out, and the remaining region of the aorta is stitched back together (sutured) [3]. Catheter based interventions are also available, and include balloon angioplasty, where a balloon on a catheter is inserted into the aorta and positioned within the coarctated region, and the balloon is then expanded. Stent placements are a modification of balloon angioplasty. In this treatment, a metal mesh surrounds the balloon, and after expansion of the balloon the mesh expands and is embedded into the aorta wall, providing additional support.

Studies that compare the long term effectiveness of the treatments have so far failed to identify suitable data sets [6]. The treatment that provides the best long term outcomes for patients remains unknown, so treatments are currently chosen based on the presentation of the disease and possible short term complications [7]. Common complications of treatments include hypertension, which is increased blood pressure, and aneurysms, which are bulges in the aorta wall that can burst and lead to death [3].

This study predicts the effects that the resection and end-to-end anastomosis treatment and stent treatment may have on patients. Since both treatments alter the aorta wall stiffness, simulations of blood flow through aortas with altered wall stiffness are analysed to predict the effects of the two treatments. 


\section{Model outline}

The aorta is a thick-walled, elastic vessel that carries blood from the heart. Blood pressure and velocity waves are generated by contraction of the heart in a heartbeat and interaction of the blood with the artery walls [8]. The contraction of the heart results in a sudden increase in the blood volume in the aorta. Since the aorta expands to accommodate this increase, here it will be assumed that blood is an incompressible fluid. Elastic energy is generated during distension and, when released, the artery contracts and a pulse wave is produced after the heartbeat.

This study uses simulations of a one dimensional (1D) mathematical model of blood flow through the aorta. The simulations were run with the Nektar1D software [9]. Full details of the model and assumptions are described by Alastruey et al. [10]. The assumptions include laminar flow, and that blood is an incompressible and Newtonian fluid.

The governing equations are derived from the conservation of mass and momentum:

$$
\begin{aligned}
\frac{\partial A}{\partial t}+\frac{\partial Q}{\partial x} & =0 \\
\frac{\partial Q}{\partial t}+\frac{\partial}{\partial x}\left(\alpha \frac{Q^{2}}{A}\right)+\frac{A}{\rho} \frac{\partial P}{\partial x} & =\frac{f}{\rho},
\end{aligned}
$$

where $x$ is the position along the aorta, $t$ is time, $A(x, t)$ is the cross-sectional area, $\mathrm{Q}(\mathrm{x}, \mathrm{t})$ is the blood volume flow rate, $\mathrm{P}$ is average blood pressure over a cross-section, $\rho$ is the density of blood, and $f(x, t)=2 \mu \pi R\left[\frac{\partial u}{\partial r}\right]_{r=R}$ is the frictional force per unit length that comes from integrating the incompressible 3D Navier-Stokes equations for axi-symmetric vessels [10], where $\mu$ is blood viscosity, $R$ is aorta radius, and $u(x, r, t)$ is the velocity of fluid particles in a cross-section [11]. The non-dimensional profile shape factor (Coriolis coefficient) for flow over a cross-section, $\alpha(x, t)=\frac{1}{\mathrm{AU}^{2}} \int_{\mathrm{A}} \mathrm{u}^{2} \mathrm{~d} \sigma$, accounts for non-linearity in $u(x, r, t)$, where $U$ is the average velocity over the crosssection and $d \sigma$ is a differential element of area. The tube law relates pressure 
and area to account for the interaction between the blood and the aorta wall [10]:

$$
P(x, t)=P_{e}(A(x, t), x)+\frac{\Gamma A_{0}}{\sqrt{A(x, t)}} \frac{\partial A}{\partial t},
$$

with

$$
\begin{aligned}
P_{e}(A(x, t), x) & =P_{e x t}+\frac{\beta(x)}{A_{0}}\left(\sqrt{A(x, t)}-\sqrt{A_{0}}\right), \\
\beta(x) & =\frac{4}{3} \sqrt{\pi} E(x) h, \quad \Gamma=\frac{2}{3} \sqrt{\pi} \psi h .
\end{aligned}
$$

Here $P_{e}$ is the elastic component of pressure, $P_{\text {ext }}$ is external (extramural) pressure, $A_{0}$ is the cross-sectional area when $P=P_{\text {ext }}, h$ is the aorta wall thickness, $E(x)$ is the Young's modulus of the aorta wall and $\psi$ is the wall viscosity. In our model the Young's modulus is varied to represent the different aorta wall stiffnesses from different treatments for CoA.

\subsection{Parameter values}

Table 1 provides parameter values used in the simulations and Figure 3 gives a schematic of the aorta cross-section along its length. This includes space and time step values used in the simulations, as well as the value for mean blood flow, $\mathrm{Q}$. The physical description of the aorta and the properties of its wall are given, along with haemodynamic parameters of the blood. The final values listed in Table 1 are the external pressure and the Windkessel parameters for a three-element Windkessel model [12], which account for compliance of the artery, the characteristic impedance of the vessel, and systemic vascular resistance.

\section{$2.2 \quad$ Stiffness}

As CoA is a disease characterised by a narrowing of the aorta, treatments of the disease alter the aorta wall and its material properties, in particular 
Table 1: Parameter values used in numerical simulations.

Parameter

Element size

Time step

Mean blood flow rate, $\bar{Q}$

Aorta length

Radius at diastolic pressure, $\mathrm{R}$

Wall thickness, $h$

Young's modulus of aorta wall (normal), E

Blood density, $\rho$

Blood viscosity, $\mu$

Wall viscosity, $\psi$

External pressure, $P_{\text {ext }}$

Windkessel R1

Windkessel C

Windkessel R2
Value (units)

$0.0025(\mathrm{~m})$

$1 \times 10^{-7}(\mathrm{~s})$

$6.17\left(\mathrm{~L} \mathrm{~min}^{-1}\right)$

$0.2413(\mathrm{~m})$

$0.012(\mathrm{~m})$

$0.0012(\mathrm{~m})$

$400(\mathrm{kPa})$

$1060\left(\mathrm{~kg} \mathrm{~m}^{-3}\right)$

$0.004(\mathrm{~Pa} \mathrm{~s})$

$186\left(\mathrm{kPa} \mathrm{s} \mathrm{mm}^{-1}\right)$

$9467(\mathrm{~Pa})$

$1.1752 \times 10^{7}\left(\mathrm{~Pa} \mathrm{~s} \mathrm{~m}^{-3}\right)[13]$

$1.0163 \times 10^{-8}\left(\mathrm{~m}^{3} \mathrm{~Pa}^{-1}\right)[13]$

$1.1167 \times 10^{8}\left(\mathrm{~Pa} \mathrm{~s} \mathrm{~m}^{-3}\right)$ [13]

Figure 3: Cross-section of model indicating the physical size of the domain and wall thickness $h$, mean blood flow rate $\bar{Q}$, Young's modulus $E(x)$ and viscosity $\psi$ of the aorta wall, and blood density $\rho$ and viscosity $\mu$.

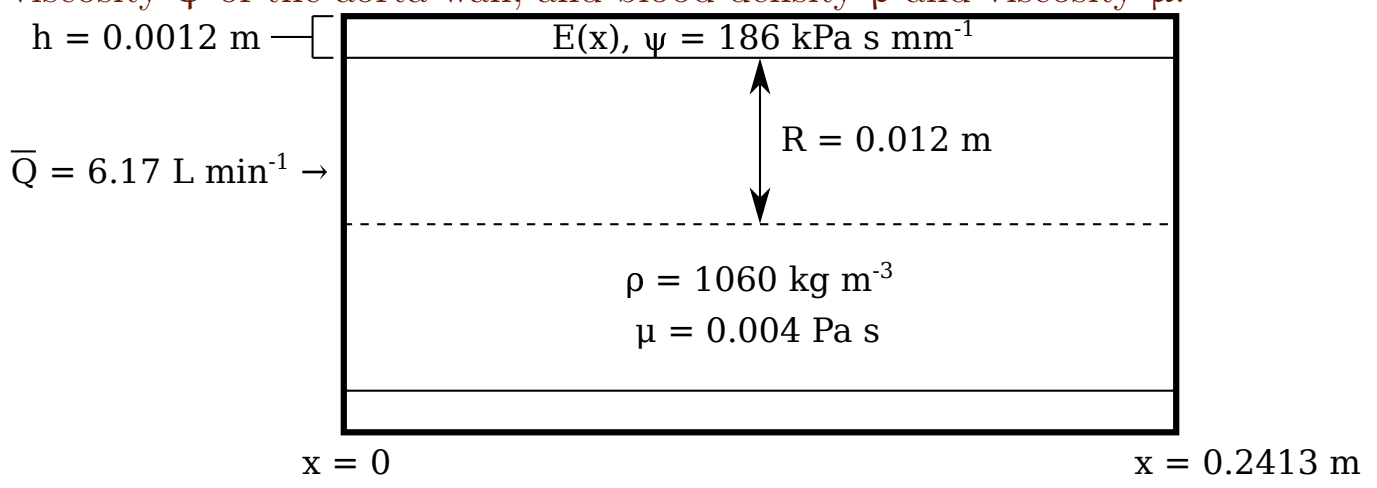


Figure 4: Stiffness for the resection and end-to-end anastomosis treatment.

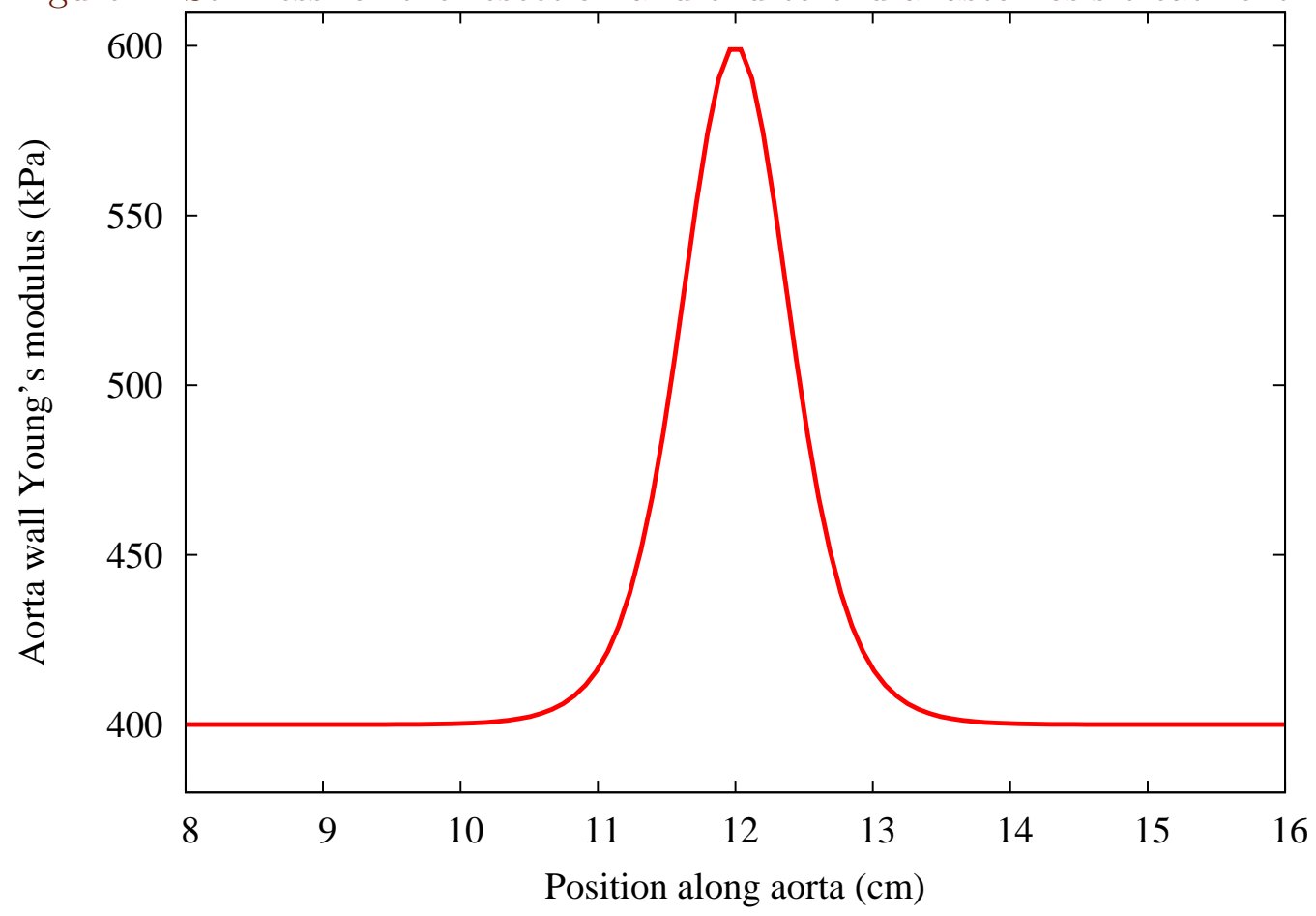

stiffness. For example, in the stitched region of the aorta following resection and end-to-end anastomosis, the suturing increases the stiffness of the artery wall in a narrow region around the anastomosis, whereas the metal mesh used for a stent increases the wall stiffness over a larger region. These altered aorta wall stiffnesses resulting from different treatments are modelled by altering the Young's modulus of the aorta wall in the affected regions. Figures 4 and 5 presents the resulting patterns of altered stiffness. 
Figure 5: Stiffness for the stent treatment.

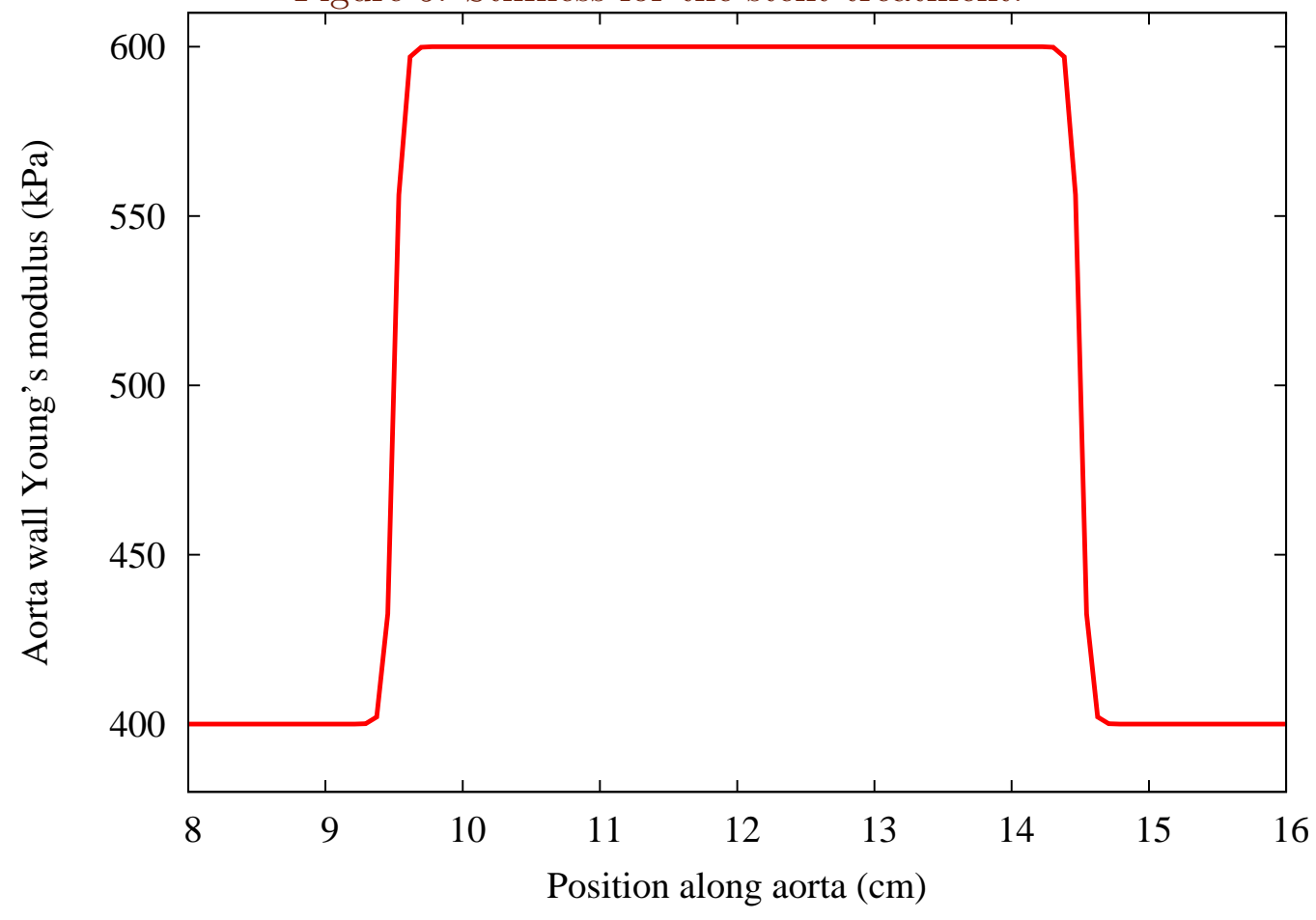

\subsection{Numerical method}

The discontinuous Galerkin (DG) method is used to solve the 1D governing equations (1) and (2). Alastruey et al. [10, App. 2] give full details of the implementation of the DG method. Briefly, the DG method is a spectral method which uses high order piecewise polynomials, such as the Legendre polynomials, as the basis functions for the solution. The residual function is then weighted with test functions, which are the same as the basis functions, and is set to zero after integration, yielding the solution.

The inlet boundary value is a high degree polynomial interpolated from recordings of human heartbeats (Figure 6 ) [14, 13, 15]. The boundary value 
Figure 6: Flow profile from a single heartbeat [13].

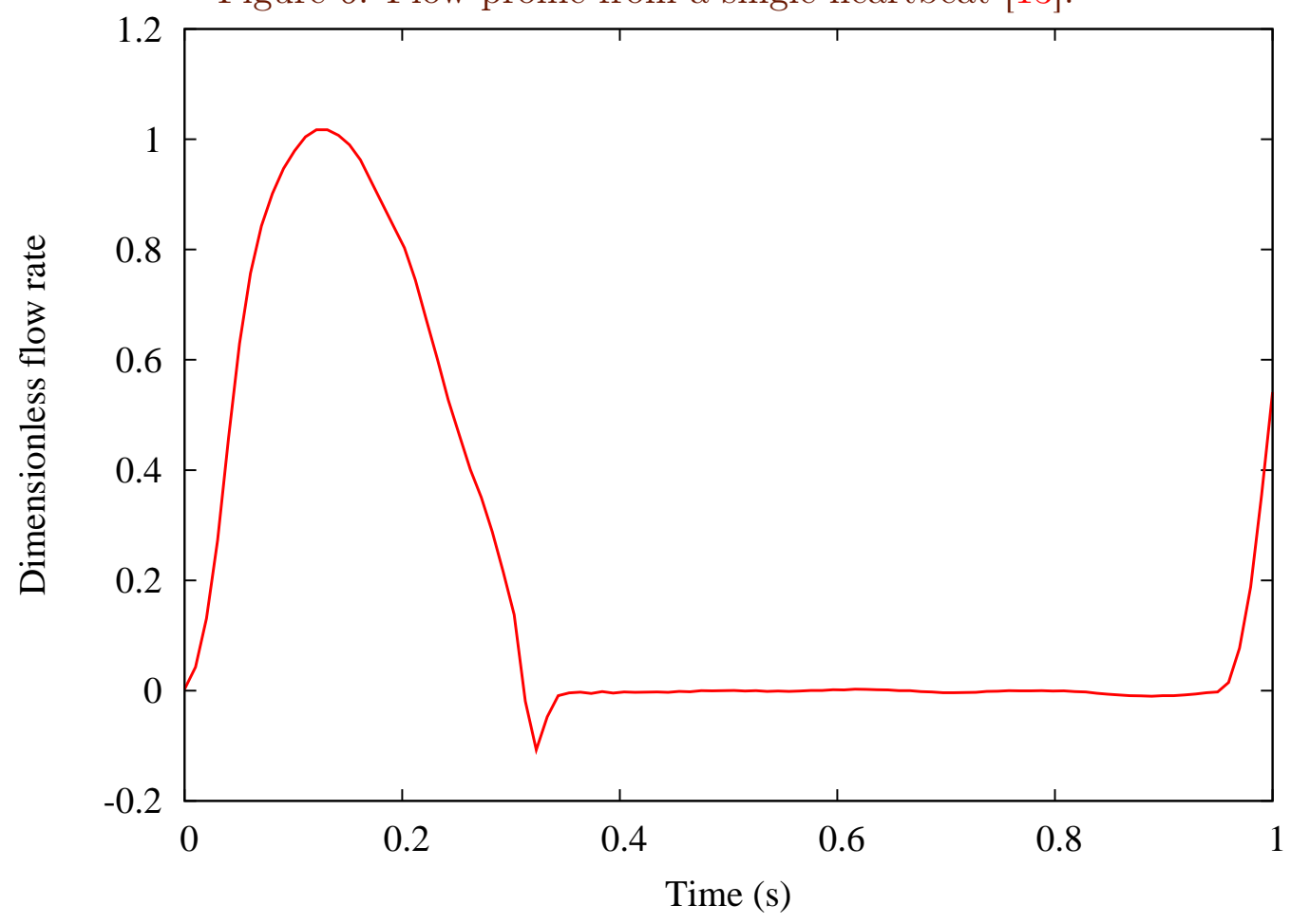

is a dimensionless flow rate. The actual flow $\mathrm{Q}$ is determined by multiplying the dimensionless flow rate by the mean flow rate $\bar{Q}$. At the outlet is a three-element Windkessel model,

$$
\mathrm{Q}\left(1+\frac{\mathrm{R}_{1}}{\mathrm{R}_{2}}\right)+\mathrm{CR}_{1} \frac{\partial \mathrm{Q}}{\partial \mathrm{t}}=\frac{\mathrm{P}_{e}-\mathrm{P}_{\mathrm{out}}}{\mathrm{R}_{2}}+\mathrm{C} \frac{\partial \mathrm{P}_{e}}{\partial \mathrm{t}},
$$

where $R_{1}$ and $R_{2}$ are systemic vascular resistance and characteristic impedance resistances in the system, $C$ is the capacitance and $P_{\text {out }}$ is the pressure required to pump blood to the extremities. Systemic vascular resistance is the resistance that needs to be overcome in order to pump blood throughout the vascular system, and characteristic impedance is the ratio of pressure to flow, assuming 
reflections of the pulse wave are negligible. The three-element Windkessel model accounts for effects in the vascular system that are analogous to capacitance and resistance in electric circuits [13]. The aorta expands as blood is pumped into it by the heart during the systolic phase of a heartbeat, then releases the stored blood during the diastole, acting like a capacitor. Alastruey et al. [10] estimated these parameters from in vivo measurements of pressure and flow rate.

\section{Results and discussion}

To study the blood flow in the coarctated aorta, the governing equations (1) and (2) were solved over several heartbeat cycles at a rate of 62 beats per minute for 20 seconds. To avoid early transient errors seen in the initial heartbeat cycles, only data from the last two heartbeat cycles were analysed. Parameter values for the simulation are listed in Table 1.

\subsection{Pressure changes}

Maximum blood pressure during the heartbeat cycles is analysed to determine whether treatments contribute to the hypertension and related complications that are commonly observed in treated patients (Figure 7). The peak-trough pattern in this pressure plot represents one heartbeat cycle. Figure 8 shows that maximum blood pressure is increased in the stent treatment, compared to both the healthy aorta and the resection and end-to-end anastomosis treatment. This increase appears marginal at $0.68 \%$; however, any increase in blood pressure can contribute to hypertension. In addition, maximum blood pressure in the resection and end-to-end anastomosis treatment is only marginally increased compared to the healthy treatment $(0.18 \%)$, suggesting that some other effect may be responsible for the hypertension and related complications seen in treated patients. 
Figure 7: Blood pressure measured at $x=22 \mathrm{~cm}$ over two normal heartbeats of the simulation compared with two treatments.

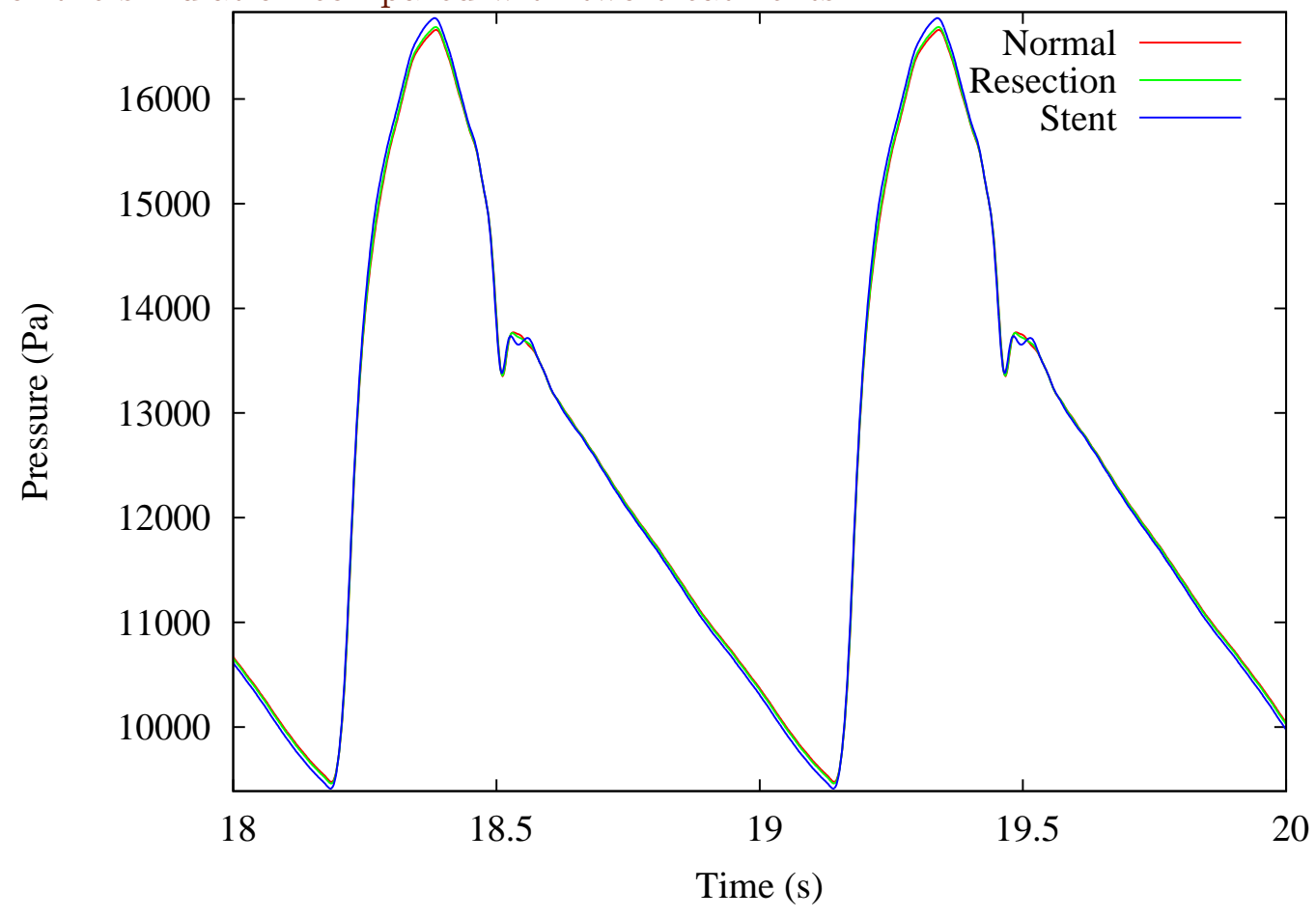

\subsection{Radial changes}

The maximum radial change is the largest change in radius in the aorta as blood is pumped through it. Radial change is analysed to determine whether the treatments contribute to aneurysms and observed complications. Figure 9 plots simulation results that show that, compared to the normal aorta, in the untreated regions, maximum radial change increases for the resection and end-to-end anastomosis treatment $(0.58 \%)$, and the stent treatment $(2.16 \%)$. The same pattern of maximum radial change is observed when the position of treatments along the aorta is changed from $6 \mathrm{~cm}$ (Figure 9) along the aorta 
Figure 8: Close up of peak blood pressures from Figure 7 for the normal and treated aortas in a heartbeat cycle.

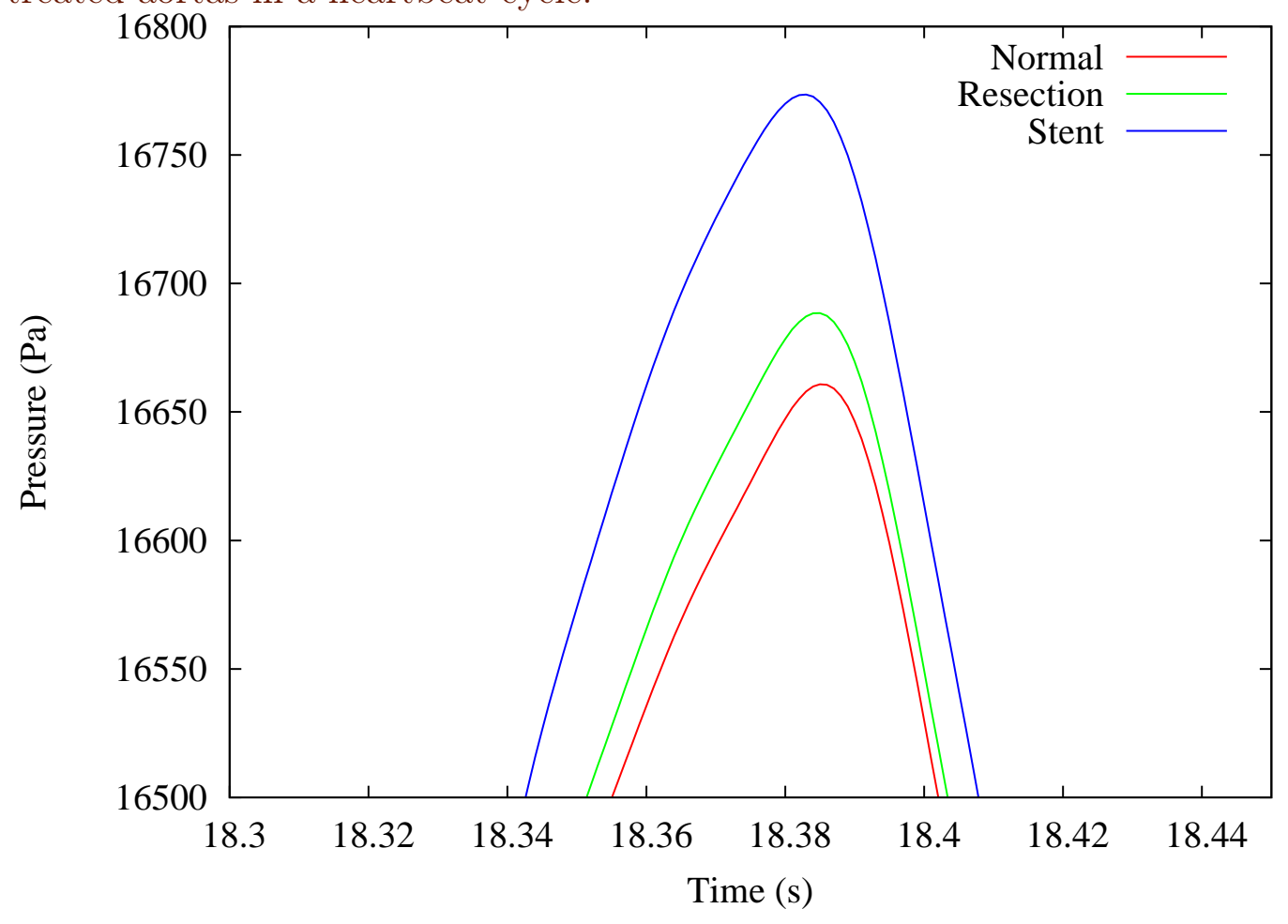

to $12 \mathrm{~cm}$ (Figure 10). The larger maximum radial change in the untreated regions for the stent treatment represents an increased expansion of the aorta with every heartbeat, compared to the resection and end-to-end anastomosis treatment and normal aorta. Increased expansion of the aorta can lead to deterioration of the aorta wall and related complications such as aneurysms. For the stent treatment, in the untreated regions, there is also an increase in maximum radial change when comparing the end of the aorta to the begining of the aorta. This increase may lead to increased deterioration of the aorta wall near its end, suggesting that the increased maximum radial change seen in the stent treatment may be compounded in this region. 
Figure 9: Maximum radial change along the normal aorta compared with treatments that are placed at $6 \mathrm{~cm}$ along the aorta.

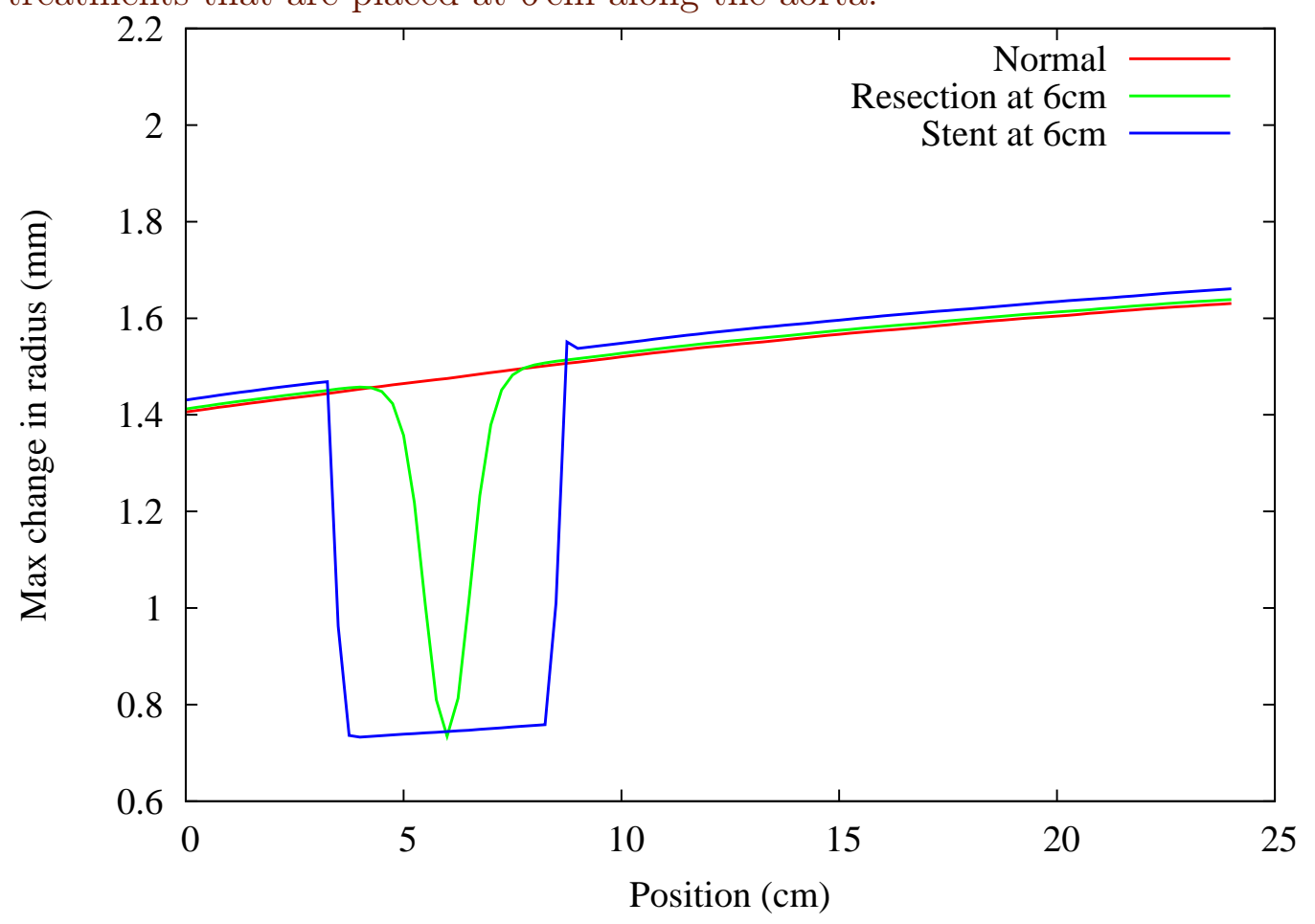

\section{Conclusion}

Life threatening conditions including hypertension and aneurysms are common complications of treatments for CoA. Little is understood about the exact cause of these complications, and there is insufficient data available to determine the best overall treatment. One factor common to all treatments is altered aorta wall material properties, including stiffness.

This study provides preliminary evidence that the hypertension and aneurysms seen in patients treated with stent placements may be due to the altered 
Figure 10: Maximum radial change along the normal aorta compared with treatments that are placed at $12 \mathrm{~cm}$ along the aorta.

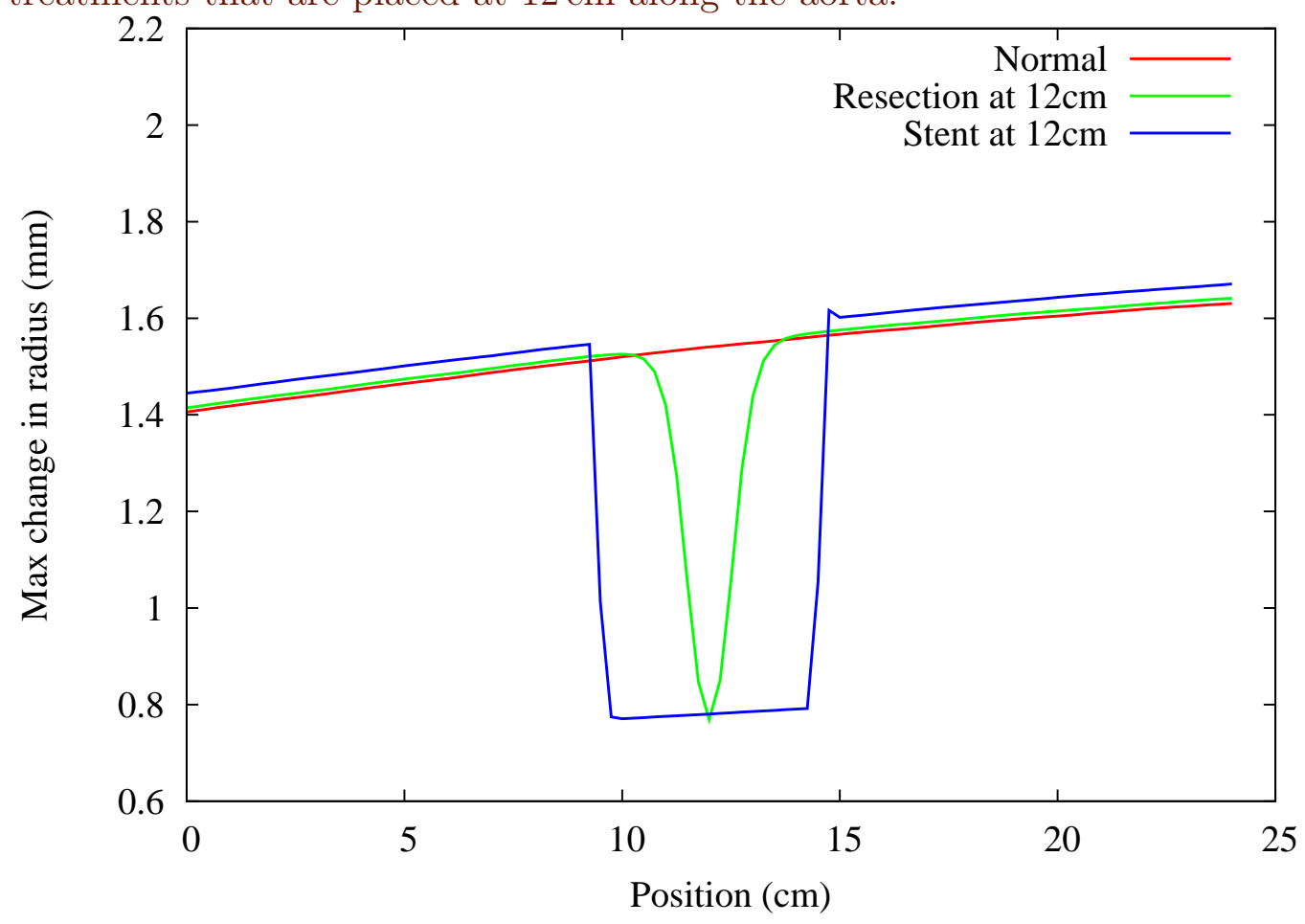

aorta wall stiffness caused by the treatment. There are weaker links between these complications and the altered aorta wall stiffness from resection and end-to-end anastomosis treatments, suggesting that the complications of hypertension and aneurysms seen in patients with this treatment may be caused by some other effect.

It was also found that there was an increase in maximum expansion of the aorta from the inlet of the aorta to its end, suggesting that treatments for CoA should be chosen based on the size and position of the coarctation, to avoid a possible compounding effect of the general increase in expansion with the increase in expansion in the regions surrounding a stent. 
The altered wall stiffness results in significant differences in the maximum aorta radial change when comparing treated regions to untreated regions, for both the resection and end-to-end anastomosis and stent treatments (Figures 9 and 10). It is worth investigating this in more detail, to determine whether there is a change in properties like wall shear stress in this region, and whether any changes can explain complications seen in treated patients.

\section{References}

[1] A. M. Rudolph, M. A. Heymann, and U. Spitznas. Hemodynamic considerations in the development of narrowing of the aorta. Am. J. Cardiol. 30(5):514-525, 1972. doi:10.1016/0002-9149(72)90042-2 C33

[2] N. S. Talner and M. A. Berman. Postnatal development of obstruction in coarctation of the aorta: role of the ductus arteriosus. Pediatrics, 56(4):562-569, 1975.

http://pediatrics.aappublications.org/content/56/4/562 C33

[3] P. S. Rao. Coarctation of the aorta. Curr. Cardiol. Rep., 7(6):425-434, 2005. doi:10.1007/s11886-005-0060-0 C33, C36

[4] Z. Keshavarz-Motamed, E. R. Edelman, P. K. Motamed, J. Garcia, N. Dahdah, and L. Kadem. The role of aortic compliance in determination of coarctation severity: Lumped parameter modeling, in vitro study and clinical evaluation. J. Biomech. 48(16):4229-4237, 2015. doi:10.1016/j.jbiomech.2015.10.017 C33

[5] M. Campbell. Natural history of coarctation of the aorta. Br. Heart J. 32(5):633-640, 1970. doi:10.1136/hrt.32.5.633 C33

[6] L. M. S. Pádua, L. C. Garcia, C. J. Rubira, and P. E. de Oliveira Carvalho. Stent placement versus surgery for coarctation of the thoracic aorta. Cochrane Db. Syst. Rev. 5:CD008204, 2012. doi:10.1002/14651858.CD008204.pub2 C36 
[7] R. Jurcut, A. M. Daraban, A. Lorber, D. Deleanu, M. S. Amzulescu, C. Zara, B. A. Popescu, and C. Ginghina. Coarctation of the aorta in adults: what is the best treatment? Case report and literature review. J. Med. Life, 4(2):189-195, 2011. http://www.ncbi.nlm.nih.gov/pmc/articles/PMC3124275/C36

[8] J. Alastruey, T. Passerini, L. Formaggia, and J. Peiró. Physical determining factors of the arterial pulse waveform: theoretical analysis and calculation using the 1-D formulation. J. Eng. Math. 77(1):19-37, 2012. doi:10.1007/s10665-012-9555-z C37

[9] J. Alastruey, M. Willemet, K. Lau, S. Epstein, and S. Vennin. Nektar1D, Haemodynamic Modelling Research Group, Kings College London, 2016. http://haemod.uk/nektar C37

[10] J. Alastruey, K. H. Parker, and S. J. Sherwin. Arterial pulse wave haemodynamics. 11th International Conference on Pressure Surges, pg. 401-442, 2012. http://wwwf .imperial.ac.uk/ssherw/spectralhp/ papers/PulseSurges_2012.pdf C37, C38, C41, C43

[11] N. P. Smith, A. J. Pullan, and P. J. Hunter. An anatomically based model of transient coronary blood flow in the heart. SIAM J. Appl. Math. 62(3):990-1018, 2002. doi:10.1137/S0036139999355199 C37

[12] N. Westerhof, F. Bosman, C. J. De Vries, and A. Noordergraaf. Analog studies of the human systemic arterial tree. J. Biomech. 2(2):121-134, 1969. doi:10.1016/0021-9290(69)90024-4 C38

[13] N. Xiao, J. Alastruey, and C. A. Figueroa. A systematic comparison between 1-D and 3-D hemodynamics in compliant arterial models. Int. J. Numer. Meth. Biomed. Eng. 30(2):204-231, 2014. doi:10.1002/cnm.2598 C39, C41, C42, C43

[14] C. A. Figueroa, I. E. Vignon-Clementel, K. E. Jansen, T. J. R. Hughes, and C. A. Taylor. A coupled momentum method for modeling blood flow 
in three-dimensional deformable arteries. Comput. Meth. Appl. Mech. Eng. 195(41-43):5685-5706, 2006. doi:10.1016/j.cma.2005.11.011 C41

[15] E. Boileau, P. Nithiarasu, P. J. Blanco, L. O. Müller, F. E. Fossan, L. R. Hellevik, W. P. Donders, W. Huberts, M. Willemet, and J. Alastruey. A benchmark study of numerical schemes for one-dimensional arterial blood flow modelling. Int. J. Numer. Meth. Biomed. Eng. 31(10):e02732, 2015. doi: $10.1002 / \mathrm{cnm} .2732$ C41

\section{Author addresses}

1. D. Pathirana, School of Natural Sciences, Griffith University, Queensland 4111, Australia. mailto:Dilan.Pathirana@griffithuni.edu.au

2. B. M. Johnston, School of Natural Sciences, Griffith University, Queensland 4111, Australia.

mailto:Barbara. Johnston@griffith. edu .au

3. P. R. Johnston, School of Natural Sciences, Griffith University, Queensland 4111, Australia.

mailto:P. Johnston@griffith.edu.au 